\title{
Kategorien diskurstraditionellen Wissens als Grundlage einer kulturbezogenen Sprachwissenschaft
}

1. Diskurstraditionenforschung und Diskursanalyse: Methode, Theorie und Haltung

Das in der Romanistik entwickelte Konzept der Diskurstradition als kultureller Leitfaden kommunikativ angemessener Textgestaltung ist ein zentraler Grundbegriff für eine kulturbezogene Sprachwissenschaft. Ein Charakteristikum dieses Konzepts ist seine große Spannweite. So können unter dem Konzept der Diskurstradition ganz unterschiedliche kulturelle Muster des Sprechens und Schreibens subsumiert werden: Zum diskurstraditionellen Wissen zählen kommunikative Gewohnheiten wie Begrüßungen oder Gesprächseröffnungen, doch wirken auch Textgattungen und Interaktionsstile als das Sprechen und Schreiben modellierende Diskurstraditionen.

Aus dieser Vielfalt des diskurstraditionellen Wissens resultiert das Desiderat einer Fokussierung des Konzepts. Derzeit wendet sich die Diskurstraditionenforschung nach einer Phase intensiver und konträrer theoretischer Diskussionen verstärkt text- und korpusbasierten Studien zu und diese empirische Wende verlangt - so scheint es zumindest - aus Gründen der Operationalisierbarkeit eine enger gefasste Begrifflichkeit. Doch bevor man Kriterien für eine begriffliche Zuspitzung sucht, ist grundsätzlich zu fragen, ob eine solche Fokussierung im Fall der Diskurstraditionen erkenntnisstiftend sein kann.

Der Leitgedanke der folgenden Ausführungen ist, dass gerade die Offenheit des diskurstraditionellen Wissens kontrastiv zur festeren Begrifflichkeit von Textsorten oder Textgattungen die eigentliche Qualität von Diskurstraditionen ausmacht. Daher benötigt das Konzept keine begriffliche Einengung, sondern vielmehr Kategorien, mit denen die unterschiedlichen Diskurstraditionen präziser als bisher verglichen und differenziert werden können.

Die besondere Qualität dieser Offenheit wird deutlich, wenn man am Begriff der Diskurstradition orientierte Forschungen mit der Diskursanalyse vergleicht, die ebenfalls eine kulturbezogene Sprachwissenschaft repräsentiert. Mein Aus- 
gangspunkt ist hier die von Andreas Gardt (2007) formulierte programmatische Standortbestimmung der (germanistischen) Diskursanalyse.1 Gardt definiert den Diskurs als sprachliche Interaktion zwischen verschiedenen sozialen und kulturellen Gruppierungen, die um ein gesellschaftlich relevantes Thema zentriert ist. Dabei wirkt der Diskurs zugleich als Repräsentation und Movens einer gesellschaftlich verankerten Interaktion: Der Diskurs bildet die prozesshafte Ausformung von Wissensformationen und Meinungen ab und treibt diese Prozesse zugleich voran (Gardt 2007: 29-30, 44). Diese Definition wird von einer programmatischen Ausrichtung flankiert, die über Inhalte und Ziele hinausgeht und die Diskursanalyse auf einer Metaebene als Methode, Theorie und Haltung bestimmt:

Die Diskursanalyse lässt sich also, je nach Perspektive, als Methode beschreiben, die aus verschiedenen Einzelschritten besteht und von bestimmten theoretischen Prämissen getragen wird, oder aber als Theorie, der bestimmte Methoden zugeordnet sind. Hinter Theorie und Methode steht zudem ein grundlegendes Verständnis des Fachs, das es rechtfertigt, von der Diskursanalyse als einer wissenschaftlichen Haltung zu sprechen. Dieses Fachverständnis begreift die Sprachwissenschaft als Kulturwissenschaft, also als eine Disziplin, die sprachliche Zusammenhänge vor dem Hintergrund philosophischer, religiöser, politischer, gesellschaftlicher, ökonomischer, technischnaturwissenschaftlicher, ästhetischer und alltagsweltlicher Zusammenhänge untersucht. (Gardt 2007: 39)

Diese Charakterisierung als Trias von Methode, Theorie und Haltung kann auf die Diskurstraditionenforschung übertragen werden, die mit der linguistischen Diskursanalyse das Credo einer als Kulturwissenschaft konzipierten Sprachwissenschaft teilt.

Diese Annäherung von romanistischer Diskurstraditionenforschung und germanistisch geprägter Diskursanalyse gründet auf den deutlichen Analogien beider Forschungsansätze. In der Romanistik sind die Diskurstraditionen das zentrale Konzept für die sprachwissenschaftliche Erfassung der kulturellen Prägung des Sprechens. Die Diskurstraditionen als kulturelles und sprachbezogenes Wissen liefern den Interaktanten den Leitfaden für ein angemessenes Sprechen in einer konkreten Kommunikationssituation. Sie sind richtungsweisend für die Auswahl geeigneter sprachlicher Mittel aus dem Repertoire der Einzelsprache und für deren (alltags-)rhetorisch wirksames Arrangement in einem (phonischen oder graphischen) Text, der die Intention des Sprechers

1 Eine Übersicht über die unterschiedlichen Richtungen und Schulen der Diskursanalyse vermitteln die Beiträge in Warnke (Hg.) 2007, die von Jürgen Spitzmüller und Ingo Warnke verfasste Einführung in die Diskurslinguistik (Spitzmüller/Warnke 2011) sowie die von Daniel Wrana u.a. (Hg.) 2014 herausgegebene interdisziplinäre Zusammenschau zur Diskursforschung. 
erfolgreich vermittelt. ${ }^{2}$ Als kulturelles Wissen werden die Diskurstraditionen von sozial und kulturell bestimmten Gruppierungen einer Gesellschaft praktiziert und tradiert. ${ }^{3}$ Eine Diskurstradition kann sich durch Übernahme auf eine größere Sprechergruppe ausdehnen und auf diese Weise den Interaktionsstil einer sozialen oder kulturellen Gemeinschaft verändern. Expandiert eine Diskurstradition oder wandert sie von einer Gruppierung zu einer anderen, dann verändert sich auch der Interaktionsstil innerhalb einer Gesellschaft. Eine solche diskurstraditionelle Expansion kann sich beispielsweise darin niederschlagen, dass Gesprächspartner in ihrem Anredeverhalten stärker als bisher auf das Duzen zurückgreifen, höfliche Bitten anders als früher üblich formulieren oder beim Erzählen von Geschichten neue narrative Techniken verwenden. Eine Modifizierung der Diskurstraditionen kann sich jedoch auch darin niederschlagen, dass gesellschaftlich relevante Themen in einer veränderten Weise diskutiert und soziale Phänomene anders als bisher thematisiert und versprachlicht werden. Der Wandel erfasst damit nicht allein die Diskurstraditionen als Wissensbestand, sondern auch den Interaktionsstil und verändert damit zugleich die sozialen und kulturellen Gruppierungen, die sich über gesellschaftlich relevante und semantisch brisante Themen austauschen.

Versteht man die Diskurstraditionen in dieser Dynamik, dann liegt der Brückenschlag zur germanistischen Analyse von Diskursen als Repräsentation und Movens einer themengebundenen sprachlichen Interaktion sehr nahe. Denn um den Verbund von Interaktionen und Texten zu beschreiben, der einen Diskurs konstituiert, erfasst die Diskursanalyse neben sprachlichen Elementen wie etwa Leitbegriffen oder Fahnenwörtern auch Muster und Techniken der Textgestaltung. Diskurse sind daher nicht allein durch ihre Thematik charakterisiert, sondern auch durch eine Affinität zu bestimmten sprachlichen Elementen und Textmustern.

Diskurstraditionenforschung und Diskursanalyse nehmen damit sehr ähnliche Perspektiven auf Sprache und Sprechen ein. Denn beide Disziplinen analysieren das Selegieren von sprachlichen Elementen sowie von Mustern und Traditionen der Textgestaltung rückgebunden an kulturelle Gruppierungen mit dem Ziel, einen Prozess der Interaktion bzw. der Meinungsbildung zu analysie-

2 Zum Konzept der Diskurstraditionen und zur Begriffsgeschichte in der Romanistik vgl. Koch 1997: 45-47, Oesterreicher 1997: 23-25, Lebsanft 2005: 30-33 und in diesem Band, Loureda 2007: 32-34, Koch 2008: 53-55, Wilhelm 2011: 125-130, Kabatek 2001: 98-100 und in diesem Band.

3 Während historische Einzelsprachen von Sprachgemeinschaften ausgeübt und an kommende Sprechergenerationen weitergegeben werden, werden Diskurstraditionen von kulturellen Gruppierungen getragen und weitergegeben, die nicht mit den durch die Einzelsprachen definierten Sprachgemeinschaften zusammenfallen. Die diskurstraditionellen Gruppierungen können Teil einer Sprachgemeinschaft sein oder aber mehrere Sprachgemeinschaften umfassen. Vgl. hierzu Lebsanft 2006: 535-537 und Schrott 2014: 29-32. 
ren. Dennoch beinhalten Diskursanalyse und Diskurstraditionenforschung verschiedene Schwerpunktsetzungen. Denn während die Diskurstraditionen einen Wissensbestand unter dem Gesichtspunkt der dynamis fokussieren, ist der Diskurs als thematische Auseinandersetzung immer die Anwendung von Wissensbeständen und damit stärker auf das Sprechen als Tätigkeit (energeia) bezogen. Die Diskursanalyse konzentriert sich daher vor allem auf die Dynamik des Sprechens als Tätigkeit im Prozess der Ausformung von Meinungen und Einstellungen, während die Diskurstraditionenforschung sich primär mit den in diese Tätigkeit eingehenden sprachlichen und kulturellen Wissensbeständen und deren Differenzierung beschäftigt. Aus diesen unterschiedlichen Fokussetzungen ergeben sich Möglichkeiten der wechselseitigen Ergänzung, die meines Erachtens bisher kaum ausgeschöpft wurden.

Überträgt man die von Gardt (2007) für die Diskursanalyse formulierte Trias von Methode, Theorie und Haltung auf die Diskurstraditionenforschung, dann ist die Theorie die ausgeprägteste Komponente. Denn das Konzept der Diskurstraditionen entstammt dem Coseriu'schen Modell der Sprachkompetenz und ist damit innerhalb eines Modells definiert, das zu Recht den Anspruch erhebt, eine umfassende linguistische Systematik der Sprache und des Sprechens zu sein.

Angesichts der theoretischen Grundlegung auf der Coseriu'schen Sprachkompetenz kann es verwundern, dass die Diskurstraditionenforschung zugleich eine „Haltung“ impliziert, da man diesen Begriff auf den ersten Blick eher theoretisch weniger fundierten Herangehensweisen an Sprache und Texte zuschreiben würde. Entscheidend ist aber, dass es dabei im Sinne Gardts (2007: 27) um ein Ensemble grundlegender Annahmen und Überzeugungen und um ein "Gerichtetsein des wissenschaftlichen Denkens" geht (ebd.), das eine Form der wissenschaftlichen Sprachreflexion charakterisiert.

Analog zur Diskursanalyse zeichnet sich auch die Diskurstraditionenforschung durch ein kulturorientiertes sprachwissenschaftliches Denken aus. So sind im Coseriu'schen System nicht allein die Diskurstraditionen ein kulturelles Wissen; vielmehr bilden alle drei Ebenen des Sprechens und die ihnen zugeordneten Wissensbestände eine „kulturelle Schicht“ des Sprechens (Coseriu 1988: 65). Damit ist die Sprachkompetenz in ihrer Gesamtheit als kulturelle Kompetenz definiert. Entscheidend für diese Zusammenführung von Sprache und Kultur ist, dass Sprechen als Tätigkeit (energeia) Traditionen und Gewohnheiten hervorbringt und auf diese Weise Kultur schafft (Coseriu 1988: 69-70). In diesem Sinne ist die Diskurstraditionenforschung in zweifacher Hinsicht per definitionem eine als Kulturwissenschaft verstandene Sprachwissenschaft.

Auch hinsichtlich der Methoden ergeben sich Berührungspunkte zwischen Diskursanalyse und Diskurstraditionenforschung. Für die Diskursanalyse beantwortet Gardt (2007) die Frage nach den maßgeblichen Methoden in zwei- 
facher Weise. Die erste Antwort ist gewissermaßen quantitativ und besteht in einer Auflistung derjenigen linguistischen Teildisziplinen bzw. der textlinguistischen und textsemantischen Verfahren, die in der Diskursanalyse am häufigsten zum Einsatz kommen (2007: 31-32). Die zweite Antwort Gardts ist dagegen qualitativ und begreift die Diskursanalyse als ein Bündel von Forschungslinien, die durch einen konstruktivistischen Ansatz charakterisiert sind und Sprache ontologisch als Mittel der Welterschließung deuten (2007: 35-36). Da Sprache damit maßgeblich ein Instrument der Bedeutungsbildung darstellt, ist das Methodeninventar der Diskursanalyse durch eine Betonung der textsemantischen Dimension und der Textgestaltung charakterisiert:

\footnotetext{
Als Methode untersucht die Diskursanalyse die semantische Dimension sprachlicher Äußerungen auf grundsätzlich allen Ebenen des Sprachsystems, mit einem Schwerpunkt auf der lexikalischen Ebene, und, jenseits der Systemebenen, mit einem zweiten Schwerpunkt auf der Ebene der Textgestaltung, nicht im Sinne einer transphrastischen Textgrammatik, sondern im Sinne eines pragmatisch-funktionalen Textverständnisses. (Gardt 2007: 35)
}

Während die lexikalisch-semantische Dimension in der Diskurstraditionenforschung weniger dominant ist, könnte der zweite Teil der Methoden-Definition auch einem Aufsatz über Diskurstraditionen entstammen, denn die Textgestaltung im Sinne einer pragmatisch-funktionalen Textualität ist das zentrale Operationsgebiet der Diskurstraditionen, die die Selegierung sprachlicher Elemente und deren Arrangement zu einer Textgestalt anleiten. Damit ist das methodische Inventar der Diskursanalyse auch für die Diskurstraditionenforschung in hohem Maße relevant.

Im Fall der Diskurstraditionen sind aus meiner Sicht zwei Methoden zu ergänzen, die auf der Historizität und Kulturalität dieses Wissensbestandes aufbauen. Diskurstraditionelle Untersuchungen gewinnen an Schärfe, wenn sie entweder eine historisch-diachrone Vertiefung einschließen oder eine kontrastive Perspektive auf verschiedene Sprachen und Kulturen beinhalten. Daher erscheint es erkenntnisstiftend, Diskurstraditionen kontrastiv zu untersuchen, sei es historisch-diachron oder komparatistisch in synchron existierenden Gemeinschaften. Im vorliegenden Beitrag werde ich daher die Kategorien diskurstraditionellen Wissens an zwei Fallbeispielen besprechen, die beide Möglichkeiten der Kontrastierung ausschöpfen: zum einen das im 19. Jahrhundert entstandene imparfait narratif als Tradition des Erzählens, zum anderen die Technik der höflichen Bitte und ihre differenzierte Verankerung in verschiedenen Kulturräumen der Gegenwart.

Die folgenden Ausführungen verstehen sich in diesem Sinne als Beitrag zu einer Diskurstraditionenforschung, die sich - frei nach Gardt - als Methode, Theorie und Haltung definiert. 
2. Die Diskurstraditionen im System der Sprachkompetenz: Klarheit und Offenheit

Grundlage für die Formulierung diskurstraditioneller Kategorien ist das Coseriu'sche Modell der Sprachkompetenz, aus dem die Diskurstraditionen ihre Definition als kulturelles und sprachbezogenes Wissen beziehen. ${ }^{4}$

Eine zentrale Frage der kulturbezogenen Sprachwissenschaft ist, welchen Prinzipien, Regeln und Traditionen die Sprecher folgen, wenn sie kommunikative Aufgaben bewältigen. ${ }^{5}$ Das Coseriu'sche Modell erschließt die linguistische Systematik dieser Regeln und Traditionen. Ausgangspunkt ist Coserius bekannte Definition des Sprechens als universelle, allgemein-menschliche Tätigkeit, die immer in bestimmten historischen Einzelsprachen erfolgt und in konkreten Kommunikationssituationen ausgeübt wird, in denen die Sprecher als Individuen agieren (Coseriu 1988: 70). Aus diesen Eigenschaften ergibt sich eine Trias von Regeln und Traditionen, die drei verschiedene Ebenen des Sprechens konstituieren. Die universelle Ebene beinhaltet die allgemein-universellen Regeln und Prinzipien des Sprechens, die - wie z. B. das Grice'sche Kooperationsprinzip - in allen Sprachen und Kulturen Gültigkeit haben. Auf der historischeinzelsprachlichen Ebene sind die idiomatischen Traditionen lokalisiert, die als sprachliches Wissen die Beherrschung einzelner Sprachen ermöglichen. Zur dritten, individuellen Ebene der Diskurse und Texte schließlich gehören die Diskurstraditionen als kultureller Leitfaden für sprachliche Interaktion in konkreten Kommunikationssituationen. ${ }^{6}$ Diskurstraditionen sind ein kulturelles, historisch wandelbares Wissen, das es den Sprechern ermöglicht, einen Diskurs oder Text der Situation angemessen zu gestalten und ihre kommunikativen Intentionen erfolgreich zu vermitteln.

Betrachtet man die Trias der Regeln und Traditionen kontrastiv, dann ergeben sich als Kriterien der Unterscheidung zum einen Historizität vs. Universalität und zum anderen Sprachlichkeit vs. Sprachbezogenheit. Hinsichtlich der Differenzierung von Historizität und Universalität verfügen allein die universellen Regeln und Prinzipien über universelle Gültigkeit, während idiomatische Traditionen und Diskurstraditionen historisch veränderlich sind und durch

4 Coseriu 1988: 70, 95-96, 121-125. Zur Rezeption dieses Modells in der Romanistik vgl. Schlieben-Lange 1983: 13-16, 138-140, Koch 1997: 45-47 und 2008: 53-54, Lebsanft 2005: 30 und in diesem Band, Kabatek 2007: 336-339 und 2011: 91-93, Wilhelm 2001: 467-470 und 2011: 125130, Schrott 2011a: 194-197, 2012: 108-109 und 2014: 8-10.

5 Zum Begriff der kommunikativen Aufgabe vgl. Fritz 1994: 178.

6 Coseriu (1988: 95-96, 121-125) bezeichnet die universellen Regeln und Prinzipien als „elokutionelles Wissen“ und die idiomatischen Traditionen als „idiomatisches Wissen“. Die Diskurstraditionen firmieren im ursprünglichen Modell als „expressives Wissen“. 
den gemeinsamen Nenner einer in der Geschichte stehenden Traditionalität verbunden sind. Das zweite wichtige Differenzkriterium ist die Frage, ob ein Wissenstyp Teil einer Sprache ist und damit sprachliches Wissen darstellt, oder ob ein Wissensbestand zwar auf Sprechen und sprachliche Interaktion bezogen ist, aber nicht zu einer historischen Einzelsprache gehört. In der Trias der Wissensbestände sind allein die idiomatischen Traditionen ein sprachliches Wissen, das die Beherrschung einer Sprache ausmacht. Dagegen sind weder allgemeine Regeln noch Diskurstraditionen Komponenten einzelner Sprachen, sie stehen vielmehr außerhalb des idiomatischen Wissens und leiten dessen Anwendung an. In diesem Sinne sind sie als Regeln und Traditionen auf Sprache und Sprechen bezogen, aber nicht Teil von Sprache(n).

Die Diskurstraditionen sind damit ein kulturelles Wissen, das als Leitfaden und Regulans für das Sprechen als Tätigkeit dient. Die Sprecher folgen den in den Diskurstraditionen gespeicherten kulturellen Normen, wenn sie aus dem sprachlichen Repertoire bestimmte Strukturen auswählen und daraus eine (mündliche oder schriftliche) Äußerung formen. Die Diskurstraditionen sind damit das kulturelle Regulans für die als Regulatum fungierenden einzelsprachlichen Mittel. ${ }^{7}$ Als Synthese ergibt sich, dass die Diskurstraditionen mit den allgemeinen Regeln das Merkmal der Sprachbezogenheit teilen, jedoch durch das Kriterium der historischen Veränderlichkeit von den Regeln der universellen Ebene geschieden sind. Die folgende Übersicht fasst die Typologie der Regeln und Traditionen zusammen:

\begin{tabular}{cccc}
\hline Ebene & universelle Ebene & $\begin{array}{c}\text { historisch- } \\
\text { einzelsprachliche } \\
\text { Ebene }\end{array}$ & $\begin{array}{c}\text { individuelle Ebene } \\
\text { der Diskurse und } \\
\text { Texte }\end{array}$ \\
\hline Wissenstypen & $\begin{array}{c}\text { allgemeine Regeln } \\
\text { und Prinzipien des } \\
\text { Sprechens }\end{array}$ & $\begin{array}{c}\text { idiomatische } \\
\text { Traditionen }\end{array}$ & $\begin{array}{c}\text { Diskurstraditionen } \\
\text { Eigenschaften }\end{array}$ allgemein-universell \\
sprachbezogen & historisch & $\begin{array}{c}\text { historisch } \\
\text { kulturell und } \\
\text { sprachbezogen }\end{array}$ \\
\hline
\end{tabular}

Während die Diskurstraditionen damit als Wissenstyp eindeutig definiert sind, bilden sie zugleich ein fuzzy concept, dessen Offenheit eine frühe, von Peter Koch (1997) getroffene Charakterisierung betont. Nach Koch beschäftigt sich

7 Zur Unterscheidung von Regulans und Regulatum vgl. Koch 2005: 231-232. 
die Sprachwissenschaft im Bereich der Diskurstraditionen mit „Textsorten, Gattungen, Stilen, rhetorischen Genera, Gesprächsformen, Sprechakten usw.“ (1997: 45) und deckt damit ein weites Spektrum traditionellen Sprechens und Schreibens ab. ${ }^{8}$ Diese Weite dokumentieren auch zwei in der Diskurstraditionenforschung prominente Beispiele: So nennt Coseriu (1988: 162, 171-172) das Sonett als Beispiel für eine einzelsprachübergreifende Diskurstradition und Koch (1997: 43f.) analysiert die „Berliner Schnauze“ als eine kulturell bestimmte Redeweise, die ebenfalls zum diskurstraditionellen Wissen zählt.

Die Vielfalt der Phänomene lässt den Begriff der Diskurstradition als einen umbrella term erscheinen, doch darf dessen große Spannweite nicht den Blick darauf verstellen, dass es sich dennoch um einen durchaus soliden Schirm handelt. Denn das einigende Band der aufgelisteten Techniken des Sprechens ist, dass sie als kulturelles Wissen das Sprechen in konkreten Kommunikationssituationen anleiten. Da diese substanzielle Definition untrennbar mit dem System der Sprachkompetenz und seinen Dimensionen verbunden ist, kann Diskurstraditionenforschung nicht losgelöst von diesem Modell funktionieren. Vielmehr muss die grundlegende Unterscheidung der drei Wissensbestände in der Diskurstraditionenforschung stets präsent sein, damit das Konzept seine analytische Schärfe behält. ${ }^{9}$

Die Offenheit der Diskurstraditionen, die als fuzzy concept Techniken des Grüßens und Bittens, Interaktionsstile wie die „Berliner Schnauze“ oder literarische Formen wie das Sonett umfassen, ist zugleich eine Schwäche und eine Stärke. So können die Diskurstraditionen aufgrund ihres weiten Spektrums leicht als heterogenes und schwer fassbares Konzept wahrgenommen werden. Die Stärke dieser fuzziness liegt jedoch darin, dass sie die Erkenntnis ermöglicht, dass auf den ersten Blick sehr verschiedene Techniken des Sprechens (bzw. Schreibens) zu ein und demselben Wissenstyp gehören. So können kommunikative Routinen wie das Eröffnen eines Gesprächs oder die Formulierung einer Bitte, aber auch eine (literarische) Textgattung als diskurstraditionelles Wissen identifiziert werden. Denn sowohl kommunikative Routinen als auch Textsorten und Textgattungen rekurrieren auf ein kulturelles Wissen, das die Aktanten darin anleitet, sprachliche Strukturen so einzusetzen, dass eine kommunikative Aufgabe - sei es die Eröffnung eines Gesprächs, sei es die Schaffung eines literarischen Kunstwerks - angemessen erfüllt werden kann. Kommunikative Routinen und (literarische) Textgattungen haben ferner gemeinsam, dass sie einzelsprachunabhängig sind. So kann eine bestimmte

8 Zur Vielfalt diskurstraditionellen Wissens vgl. auch Koch 2008: 54 und López Serena 2011: 72.

9 Die Umdeutungen des Coseriu'schen Systems der Sprachkompetenz und deren methodologische Konsequenzen fasst Lebsanft (in diesem Band) in einer kritischen Synthese zusammen. 
Tradition der Gesprächseröffnung oder des Bittens Sprecher verschiedener historischer Einzelsprachen in einem Kulturraum verbinden und eine literarische Gattung wie das Sonett kann von kulturellen Gruppierungen, die unterschiedlichen Sprachgemeinschaften angehören, übernommen und in den jeweiligen Sprachen nachgebildet werden.

Neben diesen aus dem System der Sprachkompetenz resultierenden Charakterisierungen besitzen Diskurstraditionen noch weitere grundlegende Eigenschaften, die sie - trotz der enormen Spannweite zwischen Sonett und „Berliner Schnauze" - zusammenschließen und vereinen.

Eine grundlegende Eigenschaft von Diskurstraditionen ist ihre Omnipräsenz in der Rede. So unterliegt alles Sprechen nicht allein den allgemein-universellen Regeln des Sprechens, sondern wird stets auch durch Diskurstraditionen geformt. Sie sind die Gussformen für alles Sprechen und jede verbale Interaktion. Diese Omnipräsenz der Diskurstraditionen entspricht dem Postulat der Generizität aller sprachlichen Äußerungen, das davon ausgeht, dass der Sprachgebrauch und damit auch alle Texte bzw. Textsorten und Textgattungen auf generelle Muster rückführbar sind. Allerdings kann die diskurstraditionelle Formung von Texten unterschiedlich stark sein. So sind etwa verschiedene Diskurstraditionen des Erzählens oder der Gesprächsführung in unterschiedlichem Grad kulturell geprägt und haben folglich mehr oder weniger hohe Allgemeingültigkeit für das Erzählen oder das dialogische Sprechen. Entscheidend ist jedoch, dass keine Erzählung und kein Gespräch, mögen sie auch formlos wirken, außerhalb diskurstraditioneller Prägungen stehen kann. Als kulturelle Gussformen des Sprechens leisten die Diskurstraditionen eine kulturelle und historische Spezifizierung allgemein-universeller Regeln und Prinzipien. So ist etwa der Sprecherwechsel im Dialog ein universelles Prinzip, das in einzelnen Dialogkulturen in unterschiedlicher Weise umgesetzt wird. Analog die Narration: Das Vergegenwärtigen von Vergangenem ist ein universelles Bedürfnis des an die Gegenwart gebundenen sprechenden Menschen, das jedoch in unterschiedlichen kulturellen Traditionen des Erzählens realisiert wird. Ein Exempel für eine starke diskurstraditionelle Durchformung sind literarische Textgattungen wie das bereits zitierte Sonett, deren Traditionen der Textgestaltung explizit als literarische Normen formuliert und weitergegeben werden. Ein Beispiel für eine lockere diskurstraditionelle Anleitung wäre dagegen das ungeplante, von kommunikativen Aufgaben weitgehend entlastete, freie Alltagsgespräch, das jedoch ebenfalls diskurstraditionell geformt ist und vor allem in den Phasen von Dialogeröffnung und -beendigung kulturspezifischen Normen folgt.

Die Diskurstraditionen sind damit als Wissensbestand eindeutig über die Kriterien von Sprachbezogenheit, Kulturalität und Historizität definiert. Sie liefern die kulturelle Orientierung für die Bewältigung kommunikativer Aufgaben, indem sie die Sprecher darin anleiten, aus dem Repertoire einer Einzel- 
sprache geeignete Mittel zu selegieren und zu einem (phonischen oder graphischen) Text auszuformen. Damit bilden sie auch innerhalb des kulturellen Wissens einen Traditionstyp, der durch seine Sprachbezogenheit von kulturellen Gewohnheiten, die nicht der Sprache und der Sprachverwendung gelten, getrennt ist. Die Kulturalität der Diskurstraditionen impliziert zugleich deren Historizität. Als historisches Wissen können Diskurstraditionen kontinuierlich oder diskontinuierlich überliefert sein. Ein bestimmtes diskurstraditionelles Wissen kann versiegen und dann wieder reaktiviert werden. In diesem Sinne sind Diskurstraditionen potenziell transhistorisch: Sie sind nicht an eine kontinuierliche historische Entwicklung gebunden, sondern können sich mit Unterbrechungen in der Geschichte manifestieren. Damit verbinden Diskurstraditionen die aus dem System der Sprachkompetenz resultierende Klarheit der Definition als Wissensbestand mit einer Offenheit des Blicks auf die Vielfalt diskurstraditioneller Formungen.

Die aktuelle romanistische Diskussion um das Konzept der Diskurstraditionen konzentriert sich zu einem großen Teil auf die Abgrenzung der Diskurstraditionen vom idiomatischen Wissen als dem zweiten historischen Wissensbestand. Die Relation beider Wissenstypen und ihre Unterschiede werden dabei zumeist in Anbindung an das Coseriu'sche Modell und dessen Umformungen diskutiert. Dabei schlägt sich die Frage nach den Eigenschaften beider Wissensbestände und ihrer unterschiedlichen Historizität vor allem in der Diskussion um die Verortung der Diskurstraditionen im Coseriu'schen Modell der Sprachkompetenz nieder. ${ }^{10}$

Der vorliegende Beitrag fokussiert dagegen die Kategorien diskurstraditionellen Wissens als Basis für eine vergleichend-differenzierende Beschreibung von Diskurstraditionen. Aus dem Coseriu'schen System der Sprachkompetenz ergeben sich drei Dimensionen der Kategorisierung. Die erste Dimension der Kulturalität basiert auf dem Faktum, dass es sich bei den Diskurstraditionen um ein kulturelles Wissen handelt. Die zweite Dimension folgt aus der Sprach- und Textbezogenheit dieses Wissenstyps, der in Texten gestaltend und sinnbildend wirksam wird. Da Diskurstraditionen die Gestaltung von Texten anleiten, sind sie durch die gleichen Parameter beschreibbar, die in der Textlinguistik zur Kategorisierung von Textsorten und Textgattungen angewandt werden. ${ }^{11}$ Dis-

$10 \mathrm{Zu}$ dieser Diskussion vgl. Coseriu 1988: 89-90, Koch 1997: 45 und 2008: 53, Oesterreicher 1997: 20, 23-24 und 2009: 58-59, 65-66, Wilhelm 2011: 126-128, 130 sowie Lebsanft 2005: 30-31 und in diesem Band. Zur Historizität von Einzelsprachen und Diskurstraditionen vgl. Kabatek 2001: 98 100 und in diesem Band, López Serena 2011: 62-64 sowie Lebsanft 2006: 536-538 und in diesem Band.

11 Zur Relation von Diskurstradition und Textgattung bzw. Textsorte vgl. Loureda 2007: 35-37, Koch 2008: 53-55, Kabatek 2011: 89-90, 93-94 und López Serena 2011: 61-62, 72-73. 
kurstraditionen verfügen damit über die Dimension der Textualität. Die dritte Dimension erschließt sich aus der Relation, in der die Diskurstraditionen zu den allgemeinen Regeln und Prinzipien des Sprechens, vor allem zum Kooperationsprinzip nach Grice, stehen, und erfasst die Diskurstraditionen über das Kriterium der Kooperativität. Während die erste und zweite Dimension damit eine kulturell-textlinguistische Dimension haben, gründet die dritte in einer pragmalinguistischen Perspektive auf die Regeln und Traditionen des Sprechens.

Die beiden in diesem Beitrag exemplarisch analysierten Diskurstraditionen sind das imparfait narratif als Tradition des Erzählens und die höfliche Bitte (Kannst du mir das Brot herübergeben?) als Routineformel verbaler Höflichkeit. Bei der sprachwissenschaftlichen Analyse beider Phänomene stand zunächst im Vordergrund, wie beide Techniken funktionieren und weshalb sie als Diskurstraditionen einzuordnen sind.12 Der vorliegende Beitrag baut auf diesen Studien auf und kombiniert sie mit den aus dem Modell der Sprachkompetenz abgeleiteten Dimensionen. Daher gehen in die folgenden Kategorien sowohl theoretische Überlegungen als auch phänomenbasierte Erkenntnisse ein.

Der folgende Abschnitt behandelt die genannten diskurstraditionellen Dimensionen der Kulturalität, der Textualität und der Kooperativität. In jeder der drei Dimensionen werden Kriterien für einen kohärenten Vergleich von Diskurstraditionen erarbeitet. Dabei geht es nicht um eine exhaustive Klassifizierung, die dem Wesen eines historischen Wissensbestands widersprechen würde, sondern um eine Erarbeitung kategorisierender Parameter, die es erlauben, Diskurstraditionen präziser als bisher in ihrer textgestaltenden Funktion zu beschreiben und differenzierend aufeinander zu beziehen.

\section{Kulturalität, Textualität, Kooperativität}

\subsection{Kulturalität: definitorische Setzung, Spezifizierung, Integration}

Nachdem im vorangehenden Abschnitt gemeinsame Charakteristika diskurstraditionellen Wissens dargelegt wurden, geht es im Folgenden darum, aus der Kulturalität dieses Wissens differenzierende Charakteristika zu abstrahieren.

Eine erste Kategorie diskurstraditionellen Wissens ist das Kriterium der definitorischen Setzung. So können Diskurstraditionen ein ohne explizite Festlegungen entstandenes Alltagskonzept darstellen oder aber als Tradition auf

12 Eine ausführliche Analyse des imparfait in narrativen Kontexten findet sich in Schrott $2011 \mathrm{~b}$. Zur höflichen Bitte als Diskurstradition vgl. Schrott 2014: 16-21. 
eine definitorische Norm zurückgehen. Beispiele für ein Alltagskonzept sind kommunikative Routinen und Interaktionsstile wie die „Berliner Schnauze“ oder das sogenannte „Kiezdeutsch“. Dagegen stellen literarische Textgattungen, aber auch journalistische Textsorten wie etwa Leitartikel oder Reportage eine definitorische Setzung dar und werden als Normen tradiert.

Diskurstraditionen, die keiner definitorischen Setzung unterliegen, werden meist implizit vermittelt, so dass die Sprecher sich der formenden Traditionalität nicht immer bewusst sind. Dagegen stellen definitorisch gesetzte Diskurstraditionen meist explizite Anleitungen dar, die gelehrt und gelernt werden und den Aktanten daher in jedem Fall als normhafte Tradition gegenwärtig sind. Aus dem Gegensatz von definitorischer Setzung und ohne Setzung entwickelter kommunikativer Alltagsgewohnheit folgt, dass definitorisch gesetzte Diskurstraditionen einen engeren Gebrauchsradius haben als kommunikative Gewohnheiten, die als Teil der kommunikativen Kompetenz mit dem Sprechen erlernt werden.

Ein zweites zentrales Charakteristikum ist der Grad der kulturellen Spezifizierung von Diskurstraditionen. So können Diskurstraditionen in hohem Maße an kulturelles Wissen gebunden sein und geringe Allgemeingültigkeit besitzen, sie können umgekehrt jedoch auch vergleichsweise allgemeinen Charakter haben und nur eine schwache kulturspezifische Prägung aufweisen. Diskurstraditionen mit einer stark kulturspezifischen Prägung sind meist in einem kleinen kulturellen Kreis verankert und werden nur von wenigen Sprechern beherrscht und ausgeübt: Je spezifischer eine Diskurstradition ist, umso mehr ist sie an kulturelles Vorwissen gebunden, über das nur eine vergleichsweise kleine Sprechergruppe verfügt. Wenig spezifische Diskurstraditionen dagegen werden von einer großen Sprechergruppe beherrscht, sind im Sprachgebrauch des Alltags verwurzelt, haben oft allgemeinen Charakter und können in diesem Fall universellen Regeln des Sprechens nahestehen.

Ein drittes Kriterium hebt auf das Charakteristikum ab, dass Diskurstraditionen isoliert für sich stehende Traditionen oder aber Teil eines größeren Ganzen sein können. Dieses Kriterium zielt auf den Aspekt der Integration ab. Diskurstraditionen, die für sich stehen, sind etwa Grußformeln, die ihre Funktion ohne weitere Stützung erfüllen können und eine autonom für sich funktionierende Kulturtechnik darstellen. Diskurstraditionen können jedoch auch Teil einer umfassenderen Einheit sein. Letzteres ist der Fall, wenn eine einzelne Diskurstradition Teil einer größeren diskurstraditionellen Konfiguration ist, die sich verfestigt hat und eine Textgattung oder Textsorte bildet. ${ }^{13}$ In

13 So versteht Stempel 1972: 176 Gattungen als komplexe Verbindungen kompositer Elemente zu einer „historisch-normhaften Kompatibilitätsfigur“. Zur Relation von Diskurstraditionen und 
dieser Perspektive können Textsorten und Textgattungen als historisch verfestigte Konfigurationen von Diskurstraditionen gesehen werden, die holistisch als Textmodell wahrgenommen werden. Dieses Kriterium der (Nicht-)Integration in eine größere Einheit beinhaltet auch einen unterschiedlichen Grad an Komplexität. Denn autonom für sich stehende Diskurstraditionen haben in aller Regel eine geringere Komplexität als solche, die in der Merkmalskonfiguration einer Gattung auftreten und damit als Komponente einer umfassenderen Textstruktur fungieren.

\subsection{Textualität: Bedeutungsbildung, Textstrukturen, Bezugsfelder}

Als Wissenstyp leiten Diskurstraditionen die Gestaltung von (phonisch oder graphisch) realisierten Texten an. Damit sind Diskurstraditionen immer auf die Parameter der Textualität bezogen und wirken formend auf diese ein. Im Folgenden werden drei grundlegende Bereiche von Textualität herausgegriffen: die Bedeutungsbildung in Texten, die internen Textstrukturen und die externen Bezugsfelder von Texten.

Die Bedeutungsbildung in Texten ist Gegenstand der Textsemantik, die untersucht, wie über Referenz und einzelsprachliche Bedeutung hinausgehend auf der Ebene der Texte und Diskurse der Textsinn entsteht. ${ }^{14}$ Um textsemantische Eigenschaften von Diskurstraditionen zu beschreiben, greife ich auf das Begriffspaar von „Punktualität“ und „Flächigkeit“ zurück, das Gardt $(2008,2012)$ für die Bedeutungsbildung in Texten entwickelt hat. ${ }^{15}$ Gardt unterscheidet im Bereich der Textsemantik zwischen punktueller und flächiger Bedeutungsbildung:

Punktuelle Bedeutungsbildung kommt in prototypischer Weise dadurch zustande, dass einzelne (zumeist lexikalische) Textausdrücke in einer Weise Bedeutung evozieren, dass der betreffende Ausdruck als semantisch relevant zumindest für den weiteren Kotext seines Vorkommens bewertet wird, häufig auch für eine größere Textpassage, in besonderen Fällen sogar für den ganzen Text. [...] Bei flächiger Bedeutungsbildung entsteht der semantische Effekt durch die Gesamtheit der Bedeutungsbildung mehrerer

Gattungen vgl. Aschenberg 2003: 4-6 und Kabatek 2011: 99: „Alle Gattungen bzw. Genres sind Diskurstraditionen, aber nicht alle Diskurstraditionen sind Gattungen."

14 Zur Konstituierung des Textsinns und zur Trias von Bezeichnung (Referenz), einzelsprachlicher Bedeutung und Sinn vgl. Coseriu 1988: 79: „Der Sinn, der auf der Ebene des Diskurses übermittelt wird, ist das mit dem Sagen ,Gemeinte', d. h. der besondere sprachliche Inhalt, der mittels der Bezeichnung und der Bedeutung ausgedrückt wird, der aber in einem individuellen Diskurs über beide hinausgeht und den Einstellungen, Annahmen oder Absichten des Sprechers entspricht."

15 Gardt 2008: 214-215 und 2012: 45. 
Textelemente, ohne dass ein einzelnes dieser Textelemente bereits die erst über die Gesamtfläche des Textes entstehende Bedeutung anzeigt. [...] Zu ergänzen ist, dass mit den Ausdrücken punktuell und flächig die beiden Pole eines Kontinuums bezeichnet werden, innerhalb dessen graduelle Abstufung herrscht [...]. (Gardt 2012: 45, Hervorhebungen im Original)

Während also punktuelle Bedeutungsbildung an einzelnen Textelementen und damit an eng begrenzten Textstellen erfolgt, vollzieht sich flächige Bedeutungsbildung in der Summe des Zusammenwirkens mehrerer Textelemente über einen größeren Textabschnitt hinweg. Das Begriffspaar erfasst damit zwei grundlegende Kategorien der Konstituierung von Textsinn, die auch das Wirken von Diskurstraditionen charakterisieren. Denn da Diskurstraditionen Strukturen aus dem einzelsprachlichen Repertoire selegieren und zu einem kohärenten Text zusammenfügen, haben sie auch Anteil an der Textsemantik. Die Art und Weise, wie Diskurstraditionen die Sinnbildung in Texten beeinflussen, kann durch die Unterscheidung von punktueller und flächiger Bedeutungsbildung erfasst werden. So bewirken Diskurstraditionen, die die Gestaltung einer eng begrenzten Textstelle anleiten, eine punktuelle Bedeutungsbildung, wogegen Diskurstraditionen, die einen umfassenderen Textausschnitt prägen, in Prozesse flächiger Bedeutungsbildung involviert sind.

In der Dimension der Textualität können Diskurstraditionen ferner durch die internen Textstrukturen, die sie erzeugen, und durch die textexternen Felder, in die sie einen Text einbetten, charakterisiert werden. Diese beiden Kriterien der Textualität basieren auf der Erkenntnis, dass Diskurstraditionen (verfestigte) Konfigurationen bilden können, die in ihrer Gesamtheit Textsorten und Gattungen konstituieren. Daraus folgt, dass Diskurstraditionen Texte in den gleichen textuellen Parametern beeinflussen wie Textgattungen und dass die von der Textlinguistik formulierten Parameter der Textualität und Texttypisierung auch auf Diskurstraditionen beziehbar sind. ${ }^{16}$ Zentrale Parameter sind hier zum einen die textinternen Strukturen und zum anderen die textexternen Bezugsfelder. Textintern erscheinen folgende Kriterien der Textgestaltung entscheidend: die Selegierung sprachlicher Elemente und Strukturen, die Formung der Textmuster auf den verschiedenen Ebenen der Mikro- und Makrostruktur, das illokutionäre Profil des Textes und die konzeptionelle Markierung des Textes im Kontinuum von Nähe- und Distanzsprache (vgl. Oesterreicher 1997: 20, 23f.). Neben diesen textinternen Kriterien leisten Diskurstraditionen auch eine Anbindung des Textes an externe Bezugsfelder und liefern Hinweise auf die Umfelder, in die ein Text als Exemplar einer Gattung oder Textsorte eingebettet

16 Zu den Dimensionen von Gattungen als Kriterien der Texttypisierung vgl. Raible 1980: 335, 342346 und 1996: 65-67. $\mathrm{Zu}$ den Parametern der Textsortendifferenzierung vgl. ferner Heinemann/Viehweger 1991: 133-169 und Aschenberg 2003: 4, 6-8. 
ist. So beinhalten Diskurstraditionen Affinitäten zu sozial und kulturell differenzierten Kommunikationssituationen und verorten Texte in unterschiedlichen Wissenskontexten und Diskursuniversen. Diskurstraditionen können sich grundsätzlich auf sämtliche Parameter der internen Textstruktur und der externen Bezugsfelder beziehen, sie betreffen aber im Einzelfall meist eine begrenzte Anzahl. Erwartbar ist, dass komplexere Diskurstraditionen mehr interne Strukturen und externe Bezugsfelder beeinflussen als weniger komplexe Traditionen. Die Textstrukturen und Bezugsfelder bilden damit ein Raster, das bei der Charakterisierung von Diskurstraditionen abgefragt werden kann und ein die Textualität fokussierendes Profil diskurstraditionellen Wissens liefert.

\subsection{Kooperativität: Diskurstraditionen und Maximen}

Wie einführend dargelegt, beinhaltet die Sprachkompetenz zwei Wissensbestände, die sprachbezogen sind, selbst jedoch keiner Einzelsprache angehören: die Diskurstraditionen und die allgemein-universellen Regeln und Prinzipien des Sprechens. Da beide Wissenstypen die Anwendung des idiomatischen Wissens anleiten, liegt die Frage nahe, wie beide Wissensbestände zusammenwirken und wie sie aufeinander bezogen werden können. Da die allgemein-universellen Regeln den Rahmen allen Sprechens setzen, sind die Diskurstraditionen historische und kulturelle Ausformungen dieser allgemeinen Regeln. Ein besonders wirkmächtiges allgemeines Prinzip des Sprechens ist das Grice'sche Kooperationsprinzip, auf dessen Grundlage sich die Diskurstraditionen bewegen (1989: 26). Daraus folgt, dass alle Diskurstraditionen auf das Kooperationsprinzip und seine Maximen bezogen und durch die Relation zu diesen Maximen charakterisiert werden können. Bekanntlich wird das Kooperationsprinzip als Prinzip des kommunikativen Vertrauens durch vier Maximen ausdifferenziert, die die kommunikative Angemessenheit des Sprechens hinsichtlich der Parameter von Quantität, Qualität, Relevanz (Maxim of Relation) und perspicuitas (Maxim of Manner) näher bestimmen.

Da das Kooperationsprinzip das Sprechen in kommunikativen Kontexten anleitet, wird über diese Anbindung eine pragmalinguistische Perspektive auf das diskurstraditionelle Wissen eingebracht. Die Relation zwischen Kooperationsprinzip und Diskurstradition wird durch die Art und Weise bestimmt, wie sich die jeweilige Diskurstradition an die Maximen anschließen lässt. So kann eine Diskurstradition eine Maxime erfüllen, sie kann eine Maxime aber auch (scheinbar) beugen oder brechen. Prinzipiell beziehen sich Diskurstraditionen als historische Ausformungen des Kooperationsprinzips immer auf alle vier Maximen, doch kann eine Diskurstradition auch an eine Maxime besonders eng angeschlossen sein. So wäre es etwa denkbar, dass eine Diskurstradition 
besonders stark durch die Maxime der Quantität geprägt ist und vor allem den Grad an Informativität und Redundanz in einem Text regelt. Eine weitere Möglichkeit ist etwa, dass eine Diskurstradition die Klarheit des Sprechens (oder Schreibens) fokussiert und damit in enger Anbindung zur Maxim of Manner und ihrer Leitmaxime der perspicuitas steht.

Das Kriterium der Anschließbarkeit an die Grice'schen Maximen kann als ein weiterer Parameter für die Komplexität von Diskurstraditionen gesehen werden. Aus pragmalinguistischer Sicht sind Diskurstraditionen dann wenig komplex, wenn sie eng an eine Maxime angebunden sind. Umgekehrt ist eine Diskurstradition dann hochgradig komplex, wenn sie sich nicht geradlinig aus einer Maxime ergibt, sondern vielmehr den Bruch oder die Beugung einer Maxime beinhaltet. Bezogen auf die Maxime der perspicuitas ist eine Diskurstradition, die eine klar geordnete Textgestaltung anleitet, wenig komplex, wogegen Diskurstraditionen, die arkanes Sprechen anleiten, hohe Komplexität haben.

\subsection{Grundriss einer Kategorisierung}

In den vorangehenden Abschnitten wurden verschiedene Dimensionen vorgestellt, mit denen Diskurstraditionen differenziert werden können. Während sich ein Bündel von Kriterien aus der Kulturalität dieses Traditionstyps ergibt, folgen andere Parameter aus der Textualität von Diskurstraditionen und aus ihrer pragmatischen Relation zum Grice'schen Kooperationsprinzip und seinen Maximen. Die Kategorien diskurstraditionellen Wissens lassen sich daher drei grundlegenden Dimensionen zuordnen: der Kulturalität, der Textualität und der pragmatischen Dimension als Ausformung des Grice'schen Kooperationsprinzips.

Betrachtet man den Bereich der Kulturalität, dann ergeben sich für das diskurstraditionelle Wissen drei Differenzqualitäten:

1. Eine Diskurstradition kann definitorisch gesetzt sein oder sie kann sich ohne definitorische Setzung als kommunikative Gewohnheit entwickeln.

2. Eine Diskurstradition kann in unterschiedlichem Grade kulturell spezifiziert sein. Je spezifischer eine Diskurstradition ist, umso begrenzter ist ihr Wirkungskreis und umso kleiner und geschlossener ist die kulturelle Gruppierung, die diese Diskurstradition anwendet. Je weniger spezifiziert eine Diskurstradition dagegen ist, umso weiter gesteckt ist ihr Radius und umso größer und offener ist die Gruppierung, die diese Diskurstradition praktiziert und trägt.

3. Diskurstraditionen können autonom für sich stehen oder integraler Bestandteil einer größeren, als Textmodell fungierenden Konfiguration von Diskurs- 
traditionen sein. Im letzteren Fall ist die Diskurstradition konstitutives Element einer Textsorte oder Textgattung.

Im Bereich der Textualität, die zum einen die Bedeutungsbildung im Text und zum anderen die textinternen Strukturen und die textexternen Bezugsfelder umfasst, ergeben sich zwei mögliche Differenzierungen:

1. In der Textsemantik tragen Diskurstraditionen in unterschiedlicher Weise zur Bedeutungsbildung bei und bewirken entweder eine punktuelle oder eine flächige Bedeutungsbildung im Text.

2. Diskurstraditionen formen textinterne Strukturen und betten Texte in externe Bezugsfelder ein. Durch diesen Einfluss auf textinterne Strukturen und Bezugsfelder haben sie immer auch Anteil am Profil von Textsorten und Textgattungen.

In der pragmatischen Dimension wirken die Diskurstraditionen als Anleitung zum sprachlichen Handeln. Die Diskurstraditionen leisten dabei eine kulturelle Spezifizierung des Grice’schen Kooperationsprinzips und seiner Maximen:

1. Diskurstraditionen sind kulturelle Ausformungen der vier Maximen des Kooperationsprinzips. Sie sind grundsätzlich auf alle vier Maximen beziehbar, können jedoch besonders stark an eine Maxime (Quantität, Qualität, Relevanz oder perspicuitas) gebunden sein.

2. Eine Diskurstradition kann eine Maxime erfüllen, sie kann eine Maxime jedoch auch beugen oder brechen.

Entscheidend ist, dass diese Kriterien nicht als exhaustives Raster für eine Klassifizierung gedacht sind - dies würde der Historizität und Vielfalt diskurstraditionellen Wissens nicht gerecht werden -, sondern vielmehr ein Netzwerk von differenzierenden Kategorien bilden.

\section{Eine Diskurstradition des Erzählens im Profil: das imparfait narratif}

\subsection{Die Funktionsweise des imparfait narratif}

Das imparfait narratif ist eine Diskurstradition des Erzählens, bei der das imparfait in narrativ-sukzessiven Kontexten eingesetzt wird. Das Funktionsprofil der Form illustriert das folgende Beispiel:

(1) L'île de Capraja [...] appartenait à Gênes depuis 1507. Dès qu'il eût connaissance de la faiblesse de la garnison de Capraja, Paoli hâta les préparatifs. Le 16 février 1767, un corps expéditionnaire de 200 hommes, commandé par Achille Murati, débarquait sur l'île et investissait la citadelle. Le 31 mai suivant, l'île capitulait. 
Cette défaite sonna pour Gênes le glas de son occupation de la Corse. (Guide Michelin: Corse, 101) ${ }^{17}$

Der Textausschnitt schildert die Geschichte Korsikas im 18. Jahrhundert, wobei die zeitliche Sukzession explizit durch temporale Angaben versprachlicht wird (le 16 février 1767, le 31 mai suivant). Diese narrativ-sukzessive Diskursorganisation lässt durchgehend die perfektiv markierte Form des passé simple erwarten, das begrenzte, dem Zeitfluss unterliegende Sachverhalte ausdrückt und daher für den Ausdruck von Sukzessionen prädestiniert ist.18

Wie das Beispiel zeigt, finden sich im Text die erwartbaren perfektiven passé simple-Formen, doch werden die sukzessiven Ereignisse zum Teil auch mit dem imparfait (débarquait, investissait, capitulait) versprachlicht. Diese Verwendung ist hochgradig markiert, da das imparfait als imperfektiver Aspekt Handlungen versprachlicht, deren zeitliche Begrenzungen ausgeblendet sind und die sich ,schon und noch" in einer Situation in der Vergangenheit vollziehen, in der sie zeitlich verankert sind. ${ }^{19}$ Diese "Ankersituation“ ist Teil der imperfektiven Semantik und wird vom imparfait in den Text eingebracht. ${ }^{20}$ Aufgrund seiner imperfektiven Semantik kann das imparfait keine narrativ aufeinanderfolgenden Sachverhalte versprachlichen, da die sukzessiven Ereignisse einer Erzählung immer eine zeitliche Begrenzung haben. Der imperfektive Aspekt der imparfait-Formen kontrastiert daher im Beispieltext mit der Ordnung der Sukzession und der narrativen Diskursorganisation.

Diese Verwendung des imparfait für Sachverhalte, die sinnvoll nur als narrative Sukzession verstanden werden können, ist eine seit der Mitte des 19. Jahrhunderts häufige Technik des Erzählens. Entscheidend ist, dass die Verwendung des imparfait narratif nicht auf eine Abschwächung der imperfektiven Aspektmarkierung zurückzuführen ist, sondern gerade den Kontrast zwischen kontextuell gegebener zeitlicher Begrenzung und imperfektiv-entgrenzendem Aspekt nutzt, um den Effekt einer semantischen Kollision zu erzeugen, bei der das imparfait die sukzessiven Sachverhalte gleichsam aus dem Zeitfluss heraushebt. ${ }^{21}$ Eine Variante dieser Verwendung illustriert das folgende Beispiel:

17 Guide Michelin (1985): Corse. - Clermont-Ferrand: Michelin/Propriétaires-éditeurs.

18 Zur Opposition von imparfait und perfektivem passé simple vgl. Togeby 1982: 318-319.

19 Zum Aspekt im Französischen vgl. etwa Togeby 1982: 318-319, Pollak ${ }^{2} 1988$ : bes. 117-124 und Becker 2010: 86, 92.

20 Zur Ankersituation vgl. Schrott 2011b: 141. Einen verwandten Ansatz der Situationseinbindung vertreten Berthonneau/Kleiber 1993: 63-66, 68 in ihrem Modell des imparfait als anaphorischmeronymische Form.

21 Zum imparfait narratif vgl. Togeby 1982: 341-345, Pollak ${ }^{2} 1988$ : 124-144, bes. 138, Bres 2005: 6366 und Schrott 2011b. 
(2) Mais avant qu'il [Yvars] les eût rejoints, ils se retournèrent soudain vers les portes de l'atelier qui venaient de s'entrouvrir. Ballester, le contremaître, apparaissait dans l'embrasure. Il ouvrait l'une des lourdes portes et, tournant alors le dos aux ouvriers, la poussait lentement sur son rail de fonte. (Camus: Les muets, 67) ${ }^{22}$

Auch hier beinhaltet das Handlungsgerüst eine zeitliche Abfolge: Yvars kommt an, die Arbeiter drehen sich zur Tür, Ballester erscheint im Türrahmen, öffnet einen Türflügel und schiebt ihn auf. Während der Kontext damit eine deutliche Sukzession beinhaltet, hebt das imparfait die Handlungen durch den imperfektiven Aspekt aus diesem Zeitfluss heraus und löst dadurch die Linearität der Sukzession auf.

Die Beispiele verdeutlichen, dass das imparfait narratif eine Technik des Erzählens ist, die den imperfektiven Aspekt innovativ nutzt und auf diese Weise eine neue Technik erzählerischer Darstellung erzeugt. ${ }^{23}$ Allerdings unterscheiden sich die Textbeispiele darin, dass es in (1) um eine Kollision zeitlicher Natur - Begrenztheit der Sukzession vs. Unbegrenztheit des imperfektiven Aspekts - geht, während das imparfait narratif in (2) über den zeitlichen Kontrast hinaus auch eine Perspektivierung der imperfektiv versprachlichten Sachverhalte leistet. Die Grundlage für diese durch (2) illustrierte Perspektivierung ist, dass die mit dem imparfait verhaftete Ankersituation neben der zeitlichen auch eine personale Dimension hat und einen Wahrnehmer beinhaltet, aus dessen Sicht die Ereignisse wiedergegeben werden. ${ }^{24}$ Durch diese personale Dimension präsentiert das imparfait in Beispiel (2) die Aktionen Ballesters als wahrgenommene Handlungen, wobei die Arbeiter im Text explizit als Beobachter benannt werden. Diese Rückbindung an ein wahrnehmendes Subjekt bewirkt, dass die versprachlichten Sachverhalte nicht als durchgehende Sukzession erscheinen, sondern dass die Sequenz vielmehr aus einer Reihe von Wahrnehmungsakten zusammengesetzt wird. Auf diese Weise löst das imparfait die an sich sukzessiven Sachverhalte aus ihrer narrativen Ordnung und durchbricht die Linearität des Erzählens. Die vom imperfektiven imparfait geleistete Rückbindung an ein wahrnehmendes Subjekt erzeugt eine Beobachterperspektive und betont den Sehakt. Daher ist das imparfait narratif eine Verwendung, die maßgeblichen Anteil an der perspektivierenden Erzähltechnik des Realismus hat.

22 Albert Camus: Les muets. - In: Albert Camus (1963): L'exil et le royaume. - Paris: Gallimard, Nachdruck o. J.

23 Nach Blumenthal 1986: 102-103, 107 greift eine systemlinguistische Erklärung, die das imparfait lediglich als Ersatzform für ein sich zurückziehendes passé simple sieht, zu kurz. Vielmehr wird die Ausdehnung der imparfait-Verwendung im 19. Jahrhundert auf eine Technik der „Entlinearisierung" des Erzählens zurückgeführt.

24 Vgl. Schrott 2011b: 152-154, 156-157. 
Auf diese Weise illustrieren die beiden Textausschnitte zwei Typen des imparfait narratif: einen zeitlichen Typ, der mit der zeitlichen Kollision von begrenzendem Kontext und entgrenzender Imperfektivität arbeitet, und einen perspektivierenden Typ, der über den Wahrnehmungsakt die aus dem Kontext erschließbare narrative Ordnung durchbricht und so der Linearität des Erzählens entgegenwirkt (vgl. Schrott 2011b).

\subsection{Das imparfait narratif im Profil}

\subsubsection{Kulturalität}

Als kulturelle Tradition ist das imparfait narratif ein literarisches Verfahren, das an bestimmte Gattungen und Kontexte gebunden ist. Als kulturelle Tradition mit literarischem Ursprung stellt das imparfait narratif eine Technik dar, die als Stilmittel erlernt und bewusst eingesetzt wird. Anders als das narrative Präsens ist das imparfait narratif damit keine kommunikative Praxis des sprachlichen Alltags, sondern fungiert aufgrund seiner Literarizität als definitorisch gesetzte Diskurstradition. Aus dieser definitorischen Setzung folgt auch, dass das imparfait narratif sich durch einen hohen Grad an kultureller Spezifizierung auszeichnet. Im Fall des imparfait narratif hat diese Markiertheit allerdings im Laufe der Zeit abgenommen, denn das Verfahren hat sich auf immer mehr narrative Textgattungen und Textsorten ausgedehnt und ist auch außerhalb der Literatur gebräuchlich, etwa in journalistischen Texten. Damit ist aus einer innovativen Technik narrativer Präsentation ein routiniertes Verfahren geworden, das in keiner Sportreportage fehlen darf. Doch trotz dieser Verbreitung stellt das imparfait narratif immer noch ein vergleichsweise elitäres Verfahren dar, denn die Verwendung in narrativen Kontexten ist an geschriebene, meist distanzsprachliche Texte gebunden und stellt eine Technik professionellen Schreibens dar, die nur von einer kleinen, entsprechend ausgebildeten kulturellen Gruppierung beherrscht wird.

Aufschlussreich ist im Fall des imparfait narratif ferner die Frage nach der Einbindung in ein größeres Ganzes wie etwa in eine Textgattung. Wie angedeutet, ist das imparfait narratif im Umfeld sich wandelnder Techniken und Muster des (literarischen) Erzählens zu sehen. Beide Typen des imparfait narratif gehören einem Konzept des Erzählens an, das die Linearität narrativer Diskursorganisationen lockert und auflöst. Dabei funktioniert der erste Typ innerhalb dieses Erzählkonzepts relativ autonom für sich und erzeugt meist in Kombination mit temporalen Angaben einen zeitlichen Kollisionseffekt. Anders der zweite, mit dem Wahrnehmungsakt operierende Typ, der spezifische Kontexte benötigt, die ein Beobachten thematisieren, Spuren eines Wahrneh- 
mungsakts enthalten oder in ihrer Erzähltechnik einen wahrnehmenden Beobachter suggerieren. Das perspektivierende imparfait narratif dient hier nicht allein der Entlinearisierung des Erzählens, sondern leistet zugleich eine Perspektivierung der Erzählung und stellt daher eine komplexere Diskurstradition dar als der rein auf die zeitliche Semantik abhebende Typ.

\subsubsection{Textualität}

Zunächst zum textsemantischen Wirken des imparfait narratif. Vergleicht man den zeitlichen und den perspektivierenden Typ, dann fällt jenseits der zeitlichen bzw. personalen Fokussetzung noch ein weiterer Unterschied auf, der die Mitwirkung an der Bedeutungsbildung im Text betrifft. So operiert der zeitliche Typ über den semantischen Kontrast zwischen imperfektivem Aspekt und temporalen Angaben, die die imperfektiv versprachlichte Handlung lokalisieren, eingrenzen und in die Ordnung einer Sukzession einfügen. Anders der perspektivierende Typ, der mit einem den Sehakt betonenden Erzählen einhergeht und sich damit auf umfassendere narrative Strukturen bezieht, die den (literarischen) Text und seinen Textsinn in ganzheitlicher Weise prägen.

Diese beiden textsemantischen Wirkungsweisen des imparfait narratif können durch das von Gardt $(2008,2012)$ formulierte Begriffspaar von „Punktualität" und „Flächigkeit" erfasst werden. Beim zeitlichen Typ basiert der Kontrast zwischen Aspektform und temporaler Angabe auf einzelnen, eingrenzbaren Formen im Text und ist damit punktueller Natur. Daneben gründet die zeitliche Kollision zugleich auf einer narrativen Diskursorganisation, die den Text in seiner Gesamtheit prägt und daher flächig angelegt ist. Damit ist der erste Typ des imparfait narratif dadurch charakterisiert, dass der imperfektive Aspekt zugleich in punktueller und flächiger Weise mit dem Kontext interagiert. Anders der zweite Typ: Die imparfait-Verwendung ist hier Teil einer Erzähltechnik und erzeugt eine perspektivische Erzählweise. Dieses Phänomen prägt den gesamten Text und ist daher dominant durch eine flächige Wirkungsweise charakterisiert. Während der erste Typ auf der Ebene der Textsemantik also sowohl punktuell als auch flächig funktioniert, ist der zweite Typ exklusiv flächig angelegt.

Die textinternen Strukturen und die textexternen Bezugsfelder, auf die das imparfait narratif als Diskurstradition zugreift, differenzieren dieses textsemantische Profil noch weiter. Das imparfait narratif ist textintern betrachtet ein Verfahren, das konzeptionell distanzsprachlich angelegt ist und medial überwiegend graphisch realisiert wird. In der Ordnung der Narration wirkt das imparfait als mikrostrukturelles Verfahren, das das zeitliche Relief des Textes gestaltet und dabei eine Spannung zwischen selegierter Aspektform und narrativer Diskursorganisation aufbaut. Da das imparfait narratif meist in literari- 
schen Textgattungen auftritt bzw. in Textsorten, die sich durch das imparfait narratif eine literarische Nuance geben, steht es als Diskurstradition in enger Verbindung zum Diskursuniversum der Literatur sowie zu den Wissenskontexten und kommunikativen Bedingungen, die dieses Diskursuniversum charakterisieren.

\subsubsection{Kooperativität}

Aus pragmalinguistischer Sicht ist zu klären, inwiefern das imparfait narratif als Diskurstradition an das Kooperationsprinzip und seine Maximen, insbesondere an die Maxime der perspicuitas, angebunden werden kann. Dabei ist vor allem die Submaxime der Ordnung (Be orderly) aufschlussreich, denn die ikonische Wiedergabe von Ereignissen, bei der die Reihenfolge im Text die Sukzession in der Wirklichkeit abbildet, entspricht der Klarheit schaffenden Submaxime der Ordnung. Daher ist die Wiedergabe einer Sukzession durch das passé simple eine Technik, die eine klare zeitliche Ordnung etabliert und damit der Grice'schen Maxim of Manner und ihrer Leitmaxime der perspicuitas nahe steht.

Da das imparfait narratif diese mit dem perfektiven Aspekt arbeitende Technik des Erzählens gezielt durchbricht, erscheint das imparfait narratif als eine Diskurstradition, die durch ihre entlinearisierende Wirkung die Eindeutigkeit der narrativen Diskursorganisation mindert und damit die Maxime der perspicuitas beugt bzw. unterläuft. Allerdings wird diese Beugung durch die Konventionalität dieser Aspektverwendung gemindert. Das imparfait narratif ist seit der zweiten Hälfte des 19. Jahrhunderts eine frequente Technik und wirkt daher als konventionalisierte Beugung der Submaxime Be orderly. Daher ist das imparfait in narrativen Kontexten eine Technik, die deutlich als Diskurstradition des Erzählens definiert ist und von mit literarischen Techniken vertrauten Rezipienten verlässlich identifiziert werden kann.

\section{Direktive Fragen als Tradition höflichen Bittens}

\subsection{Könnten Sie mir das bitte näher erklären? - das Funktionsprofil direktiver Fragen}

Die in der Überschrift zitierte Technik, eine Bitte an den Gesprächspartner als Frage zu formulieren, ist als Form der höflichen Aufforderung in zahlreichen Sprachen und Kulturen belegt. Wie im Folgenden zu zeigen ist, handelt es sich bei dieser Form höflichen Bittens nicht um eine Technik, die zum idiomatischen Wissen unterschiedlicher Einzelsprachen zählt, sondern um eine kultu- 
relle Diskurstradition, die in verschiedenen Sprachen und Kulturen gebräuchlich ist: 25

(3) Könntest du mir bitte das Brot herübergeben?

(4) Por favor, ¿puedes pasarme el pan?

(5) Tu pourrais me passer le pain, s'il te plaît?

Das einzelsprachliche Material für diesen Verwendungstyp sind interrogative Strukturen, die eine ausgeprägte Affinität zur Realisierung von Frageakten haben und auch bei dieser Verwendung einen Frageakt leisten, der dann vom Adressaten als höfliche Bitte interpretiert wird. In dieser Form geleistete höfliche Bitten bezeichne ich als „direktive Fragen“. ${ }^{26}$ Diese Benennung betont, dass der Sprecher einen Frageakt vollzieht, der jedoch - im Unterschied zu anderen Fragetypen wie etwa der Informationsfrage - dem Gesprächspartner signalisiert, dass er die in der Proposition versprachlichte Handlung realisieren soll. 27

Woher dieser Effekt rührt, wird deutlich, wenn man die illokutionären Profile von Aufforderungsakt und Frageakt voneinander abgrenzt. Frageakte des Typs Wann fahren wir heute zum Baden? verweisen auf ein Wissensdefizit, versprachlichen jedoch die vom Gesprächspartner erwartete Handlung des Antwortgebens nicht explizit. Im Unterschied dazu formulieren Aufforderungen ausdrücklich die vom Sprecher gewünschte Handlung (Sag' mir doch, wann wir heute zum Baden fahren).28 Anders als Aufforderungen präsupponieren Frageakte also keine Handlungsbereitschaft des Angesprochenen, sondern eröffnen durch ihre Implizitheit Handlungsoptionen. Die höfliche Wirkung direktiver Fragen leitet sich aus dieser Optionalität ab, durch die eine vom Sprecher gewünschte Handlung als (fingierte) Option präsentiert wird, die von der Entscheidung des Adressaten abhängt. ${ }^{29}$ Dabei ist diese Optionalität in den meisten Fällen keine echte Alternative, sondern Teil einer konventionalisierten

25 Diese und ähnliche Beispiele diskutiert etwa Briz 2004: 76. Zu direktiven Fragen als Diskurstradition vgl. Schrott 2014: 16-21.

26 Diese Benennung lehnt sich an einen Begriff von Escandell Vidal (1999: 3975-3978) an, die diese Verwendung als „interrogación directiva“ etikettiert.

27 Zur Konventionalisierung direktiver Fragen in den einzelnen Sprachen vgl. zum Spanischen Escandell Vidal 1999: 3975-3978, zum Französischen Kerbrat-Orecchioni 2001: 33-52, 85 und zum Englischen Brown/Levinson 1987: 132-134.

28 Die Differenzierung von Fragen und Aufforderungen wird seit Searle intensiv diskutiert. So definiert Searle $(1969: 66-67,69)$ Fragen als „requests for information“ und ordnet sie dem Sprechakttyp der „directives“"zu.

29 Searle (1979: 43-44) beschreibt Wendungen des Typs Kannst du mir das Salz herübergeben? als indirekte Sprechakte, bei denen simultan zwei Sprechakte vollzogen werden, wobei der Adressat vom, gesagten' Sprechakt auf einen anderen, gemeinten' Sprechakt schließt. 
kommunikativen Routine. ${ }^{30}$ Der Adressat kennt die Konvention und deutet den Frageakt als höfliche Einkleidung einer Aufforderung. Die direktive Frage ist damit eine Diskurstradition, die die Sprecher darin anleitet, aus dem Repertoire einer Sprache den Interrogativsatz als geeignetes Mittel zur Realisierung einer höflichen Bitte auszuwählen.

Dieser diskurstraditionelle Charakter wird besonders deutlich, wenn man direktive Fragen kontrastiv-interkulturell und historisch-diachron betrachtet. So finden sich direktive Fragen als konventionalisierte kommunikative Routinen etwa im Deutschen, Englischen, Französischen oder Spanischen der Gegenwart und sind damit sprachübergreifend in unterschiedlichen Kulturräumen verankert. ${ }^{31}$ Der kulturspezifische Charakter zeigt sich ferner darin, dass es durchaus Sprachen bzw. Kulturgemeinschaften gibt, in denen direktive Fragen weit weniger stark als Diskurstradition etabliert sind. ${ }^{32}$ Diese Kulturgebundenheit bestätigt auch die historische Höflichkeitsforschung. So findet man etwa in Textkorpora verschiedener Sprachen des Mittelalters keine direktiven Fragen. Untersuchungen zum Altspanischen, Altfranzösischen und Altenglischen zeigen, dass die Diskurstradition der direktiven Frage im Mittelalter noch keine etablierte kommunikative Routine darstellte. ${ }^{33}$ Die direktive Frage ist damit eine Diskurstradition, deren Entstehung und Ausformung in die (frühe) Neuzeit fällt und die sich vor allem seit dem 19. Jahrhundert als hochfrequente Form höflicher Volitionalität durchgesetzt hat. ${ }^{34}$ Diese Etablierung der direktiven Frage ist keine einzelsprachliche Entwicklung, sondern ein kulturelles Phänomen, das nicht an Sprachen und Sprachgemeinschaften, sondern an Kulturen und kulturelle Gruppierungen gebunden ist. Die direktive Frage als Routine höflicher Volition ist damit eine sehr weit verbreitete Diskurstradition, die Sprachen und Sprachgemeinschaften überschreitet.

30 Zum Begriff der kommunikativen Routine vgl. Coulmas 1981: 13.

31 Vgl. zum Englischen Brown/Levinson 1987: 132-134, zum Französischen Kerbrat-Orecchioni 2001: 33-52, 85 und zum Spanischen Díaz Pérez 2003: 260.

32 So ist das Muster der direktiven Frage etwa in der polnischen oder russischen Sprechergemeinschaft als kommunikative Routine deutlich weniger etabliert als etwa im Englischen oder Deutschen. Zum Polnischen vgl. Wierzbicka 1985: 165-166, 170-171. Zu „requests“ im Russischen kontrastiv zum Englischen vgl. Wierzbicka 2010: 50-53.

33 Zum Spanischen vgl. Schrott 2006 und 2012: 120-122, zum Altfranzösischen vgl. Frank 2011: 63 65, 231-232, 288-290, 389-492 und zum Englischen Kohnen 2008: 27, 30.

34 Die Etablierung der direktiven Frage als Routineformel der höflichen Bitte fällt nach Frank 2011: 173, 231-232 im Französischen in das 19. Jahrhundert. Auch im Englischen wird die Etablierung der direktiven Frage nach Culpeper/Demmen 2011: 61-62, 74-75 auf das 19. Jahrhundert datiert. 
5.2. Direktive Fragen im diskurstraditionellen Profil

\subsubsection{Kulturalität}

Zunächst zu den Kriterien der Kulturalität. Direktive Fragen sind kommunikative Gewohnheiten und als solche keine definitorisch gesetzten Diskurstraditionen. Sie werden implizit als Routinen vermittelt und sind Teil der kommunikativen Kompetenz. Zum Kriterium der Integration ist festzuhalten, dass die direktive Frage als kommunikative Routine eine für sich stehende Diskurstradition darstellt, die keiner Textgattung angehört und damit nicht Teil eines diskurstraditionellen Gefüges ist, das eine Textgattung oder Textsorte konstituiert. Allenfalls könnte man argumentieren, dass die direktive Frage dem Bereich der verbalen Höflichkeit zuzurechnen ist und in diesem Sinne mit Konstellationen höflichen Sprechens verknüpft ist

Direktive Fragen sind eine Technik, die sprachübergreifend in zahlreichen Kulturen und Sprachen verbreitet ist und innerhalb dieser Kulturgemeinschaften von nahezu allen Sprechern beherrscht wird. Es handelt sich also nicht um eine elitäre, hochgradig spezifische Diskurstradition. Die direktive Frage hat auch deshalb einen relativ hohen Grad an Allgemeinheit, da sie aufgrund ihrer Optionalität an die Maxime Give options angebunden werden kann, der in der Höflichkeitsforschung ein hoher Grad an Universalität zugebilligt wird. ${ }^{35}$ Der große Verbreitungsgrad dieser Tradition und ihre Nähe zu allgemein-universellen Prinzipien verbaler Höflichkeit dürfen jedoch nicht darüber hinwegtäuschen, dass es sich bei der direktiven Frage nicht um eine universelle Regel des Sprechens, sondern um eine kulturelle Diskurstradition handelt. Das Fallbeispiel der direktiven Frage belegt so, dass sich die Kulturalität einer Technik des Sprechens oft erst aus kontrastiver Perspektive erschließt.

\subsubsection{Textualität}

Im Bereich der Textsemantik und der Bedeutungsbildung ist beim Kriterium der Punktualität bzw. Flächigkeit zu berücksichtigen, dass direktive Fragen als Diskurstradition einen Sprechakt anleiten und bewirken, dass ein Frageakt so realisiert wird, dass der Adressat auf eine höfliche Bitte schließt. Das Verfahren bezieht sich damit im Gesprächsverlauf zunächst auf einen einzelnen Sprechakt.

35 Das Konzept der Optionalität hat in der Höflichkeitsforschung Tradition. So nennt Lakoff 1973: 298 bei den „rules of politeness“ die Maximen „Don't impose“ und „Give options" und Leech 1983: 132 führt als eine Dimension der universell angesetzten „tact maxim“ das Geben von Optionen an. 
Berücksichtigt man die grundsätzliche Dialogizität von Sprechakten, dann umfasst das Verfahren ein Adjazenzpaar, das aus dem als Bitte fungierenden initiativen Frageakt und der Antwortreaktion besteht. Damit ist die direktive Frage eine im Dialog auf ein Adjazenzpaar beschränkte Diskurstradition, die den Dialogverlauf punktuell formt und keine flächige Gestaltung größerer Dialogabschnitte leistet. Legt man das Kriterium der Strukturen und Bezugsfelder des Textes an, dann betrifft die direktive Frage in der textinternen Struktur sehr ausgeprägt die Selegierung sprachlicher Mittel, da dieser Fragetyp exklusiv mit interrogativen Strukturen vollzogen wird. Ferner ist die direktive Frage an den Sprechakt der Frage gebunden, mit der Besonderheit, dass diese Frage als högliche Bitte funktioniert. Bei den externen Bezugsfeldern fordert die direktive Frage als Diskurstradition eine dialogische Kommunikationssituation und eine soziale Konstellation, die einen höflichen Interaktionsstil nahelegt. Die direktive Frage gehört als kommunikative Routine dem Diskursuniversum des sprachlichen Alltags an, in dem sie als ein Verfahren verbaler Höflichkeit fungiert, das im sozialen Gefüge der Interaktanten Respekt vor dem Freiraum des anderen bekundet und als Mittel der Konfliktvermeidung dient.

\subsubsection{Kooperativität}

Als kommunikative Gewohnheit ist die direktive Frage eine Diskurstradition, bei der die pragmalinguistische Dimension besonders deutlich hervortritt. $\mathrm{Zu}$ klären ist wiederum, in welcher Relation die direktive Frage zu den einzelnen Maximen des Grice'schen Kooperationsprinzips steht. Im vorliegenden Fall bietet sich insbesondere eine Bezugsetzung zur Maxim of Manner und ihrer Leitmaxime der perspicuitas an. Da bei direktiven Fragen die vom Sprecher gewünschte Handlung nicht explizit genannt wird, sondern vom Adressaten inferiert werden muss, erscheint diese Technik des höflichen Bittens zunächst als Bruch mit der von der Maxime geforderten Klarheit der Rede. Allerdings ist hier zu bedenken, dass direktive Fragen als kommunikative Routine hochgradig konventionalisiert sind und auch sprachlich in Syntax und Semantik deutlich signalisiert werden. So werden direktive Fragen exklusiv mit interrogativen Strukturen realisiert und enthalten eine Semantik des Wollens und Könnens, die explizit auf Disposition und Einstellung des Adressaten Bezug nimmt. Aus diesem Grund beinhalten direktive Fragen zwar eine Beugung der Maxime, verfügen jedoch dank der starken Konventionalisierung über hohe Eindeutigkeit und Klarheit und weisen daher keine wirkliche Ambiguität auf. 


\section{Das Konzept Diskurstradition - ein Fazit}

Diskurstraditionen haben als Konzept gegenüber anderen Modellen textlinguistischer Beschreibung zwei Vorteile. Der erste Vorteil betrifft die klare Definition des diskurstraditionellen Wissens in Absetzung von anderen in Diskurse und Texte eingehenden Wissensbeständen. Die Formel vom sprachbezogenen und kulturellen Wissen liefert eine epistemische Wasserscheide, die für die kulturbezogene Sprachwissenschaft über die Romanistik hinaus modellbildend wirken kann.

$\mathrm{Zu}$ dieser Klarheit der Definition kommt zweitens der Vorteil einer großen Offenheit des Konzepts. Diskurstraditionen können unterschiedlichen Umfang haben, sie können definitorisch als Muster der Textgestaltung gesetzt sein wie im Fall literarischer Formen oder sie können sich ohne definitorische Setzung als kommunikative Gewohnheiten entwickelt haben wie die direktive Frage. Diese Offenheit des Konzepts ermöglicht es, kulturelle Techniken der Textgestaltung filigraner zu beschreiben als dies in vielen Fällen durch Textsorten und Textgattungen möglich ist. Die aufgezeigten Möglichkeiten, diskurstraditionelles Wissen in den Beschreibungsdimensionen von Kulturalität, Textualität und Kooperativität zu erfassen, belegen, dass Diskurstraditionen sich trotz ihrer Offenheit und fuzziness differenzierenden Beschreibungen keinesfalls verweigern.

Eine weitere, darauf aufbauende Möglichkeit, Diskurstraditionen fassbarer und vergleichbarer zu machen, besteht darin, die Parameter der Kulturalität, Textualität und Kooperativität als Komponenten diskurstraditioneller Komplexität zu verstehen. Auf diese Weise werden die drei Dimensionen von der Metakategorie der Komplexität überwölbt.

Im Bereich der Kulturalität sind definitorisch gesetzte Diskurstraditionen in aller Regel komplexer als Traditionen, die sich ohne explizite Definition als Gewohnheiten im sprachlichen Alltag entwickelt haben. Auch der Grad der kulturellen Spezifizierungen kann an die Kategorie der Komplexität rückgebunden werden: Je spezifischer eine Diskurstradition ist, umso höher ist ihre Komplexität. Schließlich kann auch das Charakteristikum, ob eine Diskurstradition autonom für sich steht oder integraler Bestandteil einer Textgattung ist, als eine Form von Komplexität gedeutet werden. So ist eine Diskurstradition, die sich als Komponente einer Gattung in eine textuelle Konfiguration einfügt, aufgrund dieser Teil-Ganzes-Beziehung durch größere Komplexität charakterisiert als eine für sich stehende Diskurstradition. Wendet man diese Graduierungen der Komplexität auf das imparfait narratif an, dann besitzt es aufgrund seiner definitorischen Setzung, seiner hohen Spezifizierung und seiner Anbindung an literarische Textgattungen und Erzählformen eine hohe diskurstraditionelle Komplexität. Dagegen verfügt die direktive Frage als weit verbreitete 
kommunikative Routine, die sich ohne definitorische Setzung entwickelt hat und nicht an größere Texteinheiten gebunden ist, über einen deutlich niedrigeren Grad an Komplexität.

Auch im Bereich der Textualität kann das Kriterium der Komplexität Anwendung finden. So besitzen Diskurstraditionen, die eine flächige Bedeutungsbildung anleiten, größere Komplexität als Diskurstraditionen, die punktuell zum Textsinn beitragen. Was die internen Strukturen und externen Bezugsfelder von Texten angeht, so bietet sich ein quantifizierender Zugang an. Eine plausible Hypothese ist, dass die Komplexität einer Diskurstradition mit der Zahl der Textstrukturen und Bezugsfelder steigt, auf die sie Einfluss nimmt. Auch hinsichtlich dieses Kriteriums erweist sich das imparfait narratif als komplexe Diskurstradition. Zum einen bewirken die beiden Typen des imparfait narratif eine komplexe, flächige Bedeutungsbildung, wobei der zeitliche Typ sowohl flächig als auch punktuell operiert, wogegen der perspektivierende Typ allein in der Fläche bedeutungsbildend wirkt. Zum anderen greift das imparfait narratif einschneidend und auf verschiedenen Ebenen in die Diskursorganisation erzählender Texte ein. Die direktive Frage dagegen hat als auf einen Sprechakt begrenztes, punktuelles Verfahren der Sinnerzeugung eine geringe Komplexität. Da diese Diskurstradition zudem nur wenige, einfache sprachliche Strukturen selegiert und im weiten Feld verbaler Höflichkeit wenig spezifische Kontexte evoziert, ist sie auch hinsichtlich textinterner Strukturen und externer Bezugsfelder durch eine vergleichsweise niedrige Komplexität charakterisiert.

Schließlich können auch in der pragmatischen Dimension der Kooperativität unterschiedliche Grade an Komplexität angesetzt werden. So kann man als ersten Schritt ein quantitatives Kriterium aufstellen und annehmen, dass die Komplexität einer Diskurstradition mit der Zahl der Maximen steigt, mit denen sie verknüpft werden kann. Aussagekräftiger als diese quantifizierende Herangehensweise ist jedoch ein qualitatives Kriterium, das die Art und Weise, in der eine Diskurstradition sich auf eine Maxime bezieht, thematisiert. So erscheint es plausibel, dass Diskurstraditionen, die eine Maxime erfüllen, weniger komplex sind als Diskurstraditionen, die eine oder mehrere Maximen beugen oder brechen. Im Fall des imparfait narratif bietet sich vor allem die Anknüpfung an die Leitmaxime der perspicuitas an. Das imparfait narratif erscheint in dieser Sichtweise als eine Verwendung, die die Ordnung der Sukzession auflöst und damit die Submaxime Be orderly zumindest beugt. Für die direktive Frage kann ebenfalls eine Beugung der Maxim of Manner angesetzt werden, da die Intention nicht direkt versprachlicht wird, sondern erst inferiert werden muss und daher weniger klar zu Tage tritt als in einer (imperativischen) Volition. In diesem Sinne stellen beide Diskurstraditionen eine Beugung der Maxime dar und sind Kandidaten für eine hohe diskurstraditionelle Komplexität. Allerdings werden diese Beugungen durch die Konventionalität beider Verfahren gemin- 
dert. Dabei ist allerdings die direktive Frage verglichen mit dem imparfait narratif die deutlich stärker konventionalisierte und weiter verbreitete Technik und verfügt daher über einen geringeren Grad an Komplexität. Damit ergibt sich als Ausblick aufbauend auf Kulturalität, Textualität und Kooperativität als Metakategorie das Kriterium der Komplexität, das als Kontinuum vor allem für vergleichende Beschreibungen diskurstraditioneller Techniken nützlich ist. Über die Metakategorie der Komplexität könnten die Diskurstraditionen dann ihrerseits wieder mit dem idiomatischen Wissen verbunden werden, da der Parameter der Komplexität auch in Syntax und Semantik als Beschreibungskriterium genutzt wird.

Die erarbeiteten Kriterien machen deutlich, dass die Kategorisierung diskurstraditionellen Wissens keine mission impossible ist. Die Findung dieser Kategorien setzt allerdings voraus, dass über das Coseriu'sche System hinaus weitere linguistische Modelle einbezogen werden. So entstammen die Kategorien der Textualität und der Textsemantik der Textlinguistik und der linguistischen Diskursanalyse und das Kriterium der Kooperativität leitet sich aus der Pragmalinguistik her. Damit ist dieser Beitrag auch ein Plädoyer für eine Diskurstraditionenforschung, die sich ihrer eigenen wissenschaftlichen Tradition bewusst ist, sich jedoch zugleich stärker und reflektierter als bisher vor allem der Diskurslinguistik und der linguistischen Pragmatik öffnet, mit denen sie trotz aller Unterschiede in Theorie und Methode - eine grundlegende Haltung verbindet: das Verständnis der Sprache als Kulturobjekt und das daraus resultierende Engagement für eine kulturbezogene Sprachwissenschaft.

\section{Bibliographie}

Aschenberg, Heidi (2003): Diskurstraditionen - Orientierungen und Fragestellungen. - In: Heidi Aschenberg, Raymund Wilhelm (Hg.): Romanische Sprachgeschichte und Diskurstraditionen, 1-18. Tübingen: Narr.

Becker, Martin (2010): Die Ingredienzien des romanischen Imperfekts. - In: Linguistische Berichte 221, 79-108.

Berthonneau, Anne-Marie/Kleiber, Georges (1993): Pour une nouvelle approche de l'imparfait: l'imparfait, un temps anaphorique méronomique. - In: Langages 112, 55-73.

Blumenthal, Peter (1986): Vergangenheitstempora, Textstrukturierung und Zeitverständnis in der französischen Sprachgeschichte. - Tübingen: Niemeyer.

Bres, Jacques (2005): L'imparfait dit narratif. - Paris: CNRS Éditions.

Briz, Antonio (2004): Cortesía verbal codificada y cortesía verbal interpretada en la conversación. - In: Diana Bravo, Antonio Briz (Hg.): Pragmática sociocultural: estudios sobre el discurso de cortesía en español, 67-94. Barcelona: Ariel.

Brown, Penelope/Levinson, Stephen C. (1987): Politeness. Some Universals in Language Usage. - Cambridge: Cambridge University Press. 
Coseriu, Eugenio (1988): Sprachkompetenz. Grundzüge der Theorie des Sprechens. Bearbeitet und herausgegeben von Heinrich Weber. - Tübingen: Francke.

Coulmas, Florian (1981): Routine im Gespräch. Zur pragmatischen Fundierung der Idiomatik. - Wiesbaden: Akademische Verlagsgemeinschaft Athenaion.

Culpeper, Jonathan/Demmen, Jane (2011): Nineteenth-Century English Politeness. Negative Politeness, Conventional Indirect Requests and the Rise of the Individual Self. - In: Journal of Historical Pragmatics 12, 49-81.

Díaz Pérez, Francisco Javier (2003): La cortesía verbal en inglés y en español. Actos de habla y pragmática intercultural. - Jaén: Universidad de Jaén.

Escandell Vidal, María Victoria (1999): Los enunciados interrogativos. Aspectos semánticos y pragmáticos. - In: Ignacio Bosque, Violeta Demonte (Hg.): Gramática descriptiva de la lengua española. Madrid: Espasa, Bd. II, 3929-3991.

Frank, Birgit (2011): Aufforderung im Französischen. Ein Beitrag zur Geschichte sprachlicher Höflichkeit. - Berlin/New York: De Gruyter.

Fritz, Gerd (1994): Grundlagen der Dialogorganisation. - In: Gerd Fritz, Franz Hundsnurscher (Hg.): Handbuch der Dialoganalyse, 177-201. Tübingen: Niemeyer.

Gardt, Andreas (2007): Diskursanalyse. Aktueller theoretischer Ort und methodische Möglichkeiten. - In: Ingo Warnke (Hg.): Diskurslinguistik nach Foucault. Theorie und Gegenstände, 28-52. Berlin/New York: De Gruyter.

Gardt, Andreas (2008): Kunst und Sprache. Beobachtungen anlässlich der documenta 12. In: Achim Barsch u. a. (Hg.): Literatur - Kunst - Medien. Festschrift für Peter Seibert zum 60. Geburtstag, 201-224. München: Meidenbauer.

Gardt, Andreas (2012): Textanalyse als Basis der Diskursanalyse. Theorie und Methoden. In: Ekkehard Felder (Hg.): Faktizitätsherstellung in Diskursen. Die Macht des Deklarativen, 29-55. Berlin/Boston: De Gruyter.

Grice, Herbert Paul (1989): Logic and Conversation. - In: ders.: Studies in the Way of Words, 22-40. Cambridge, Mass./London: Harvard University Press (zuerst in: Peter Cole, Jerry L. Morgan [Hg.] [1975]: Syntax and Semantics, Bd. III Speech Acts, 41-58. New York: Academic Press).

Heinemann, Wolfgang/Viehweger, Dieter (1991): Textlinguistik. Eine Einführung. - Tübingen: Niemeyer.

Kabatek, Johannes (2001): ¿Cómo investigar las tradiciones discursivas medievales? El ejemplo de los textos jurídicos castellanos. - In: Daniel Jacob, Johannes Kabatek (Hg.): Lengua medieval y tradiciones discursivas en la Península Ibérica: descripción gramatical - pragmática histórica - metodología, 97-132. Frankfurt a. M./Madrid: Vervuert/Iberoamericana.

Kabatek, Johannes (2007): Las tradiciones discursivas entre conservación e innovación. - In: Rivista di Filologia e Letterature Ispaniche 10, 331-345.

Kabatek, Johannes (2011): Diskurstraditionen und Genres. - In: Sarah Dessì Schmid u. a. (Hg.): Rahmen des Sprechens. Beiträge zu Valenztheorie, Varietätenlinguistik, Kreolistik, Kognitiver und Historischer Semantik. Peter Koch zum 60. Geburtstag, 89-100. Tübingen: Narr.

Kabatek, Johannes (2015): Warum die „Zweite Historizität“ eben doch die zweite ist - von der Bedeutung von Diskurstraditionen für die Sprachbetrachtung. - In: Franz Lebsanft, Angela Schrott (Hg.): Diskurse, Texte, Traditionen. Modelle und Fachkulturen in der Diskussion. Göttingen: Vandenhoeck \& Ruprecht/Bonn University Press [in diesem Band]. 
Kerbrat-Orecchioni, Catherine (2001): Les actes de langage dans le discours. Théorie et fonctionnement. - Paris: Nathan.

Koch, Peter (1997): Diskurstraditionen: zu ihrem sprachtheoretischen Status und ihrer Dynamik. - In: Barbara Frank, Thomas Haye, Doris Tophinke (Hg.): Gattungen mittelalterlicher Schriftlichkeit, 43-79. Tübingen: Narr.

Koch, Peter (2005): Sprachwandel und Sprachvariation. - In: Angela Schrott, Harald Völker (Hg.): Historische Pragmatik und historische Varietätenlinguistik in den romanischen Sprachen, 229-254. Göttingen: Universitätsverlag Göttingen.

Koch, Peter (2008): Tradiciones discursivas y cambio lingüístico: el ejemplo del tratamiento vuestra merced en español. - In: Johannes Kabatek (Hg.): Sintaxis histórica del español y cambio lingüístico: Nuevas perspectivas desde las Tradiciones Discursivas, 53-87. Frankfurt a. M./Madrid: Vervuert/Iberoamericana.

Kohnen, Thomas (2008): Directives in Old English: Beyond politeness? - In: Andreas H. Jucker, Irma Taaavitsainen (Hg.): Speech Acts in the History of English, 27-44. Amsterdam/ Philadelphia: Benjamins.

Lakoff, Robin (1973): The Logic of Politeness; or: Minding your P's and Q's. - In: CLS 9: Papers from the Ninth Regional Meeting of the Chicago Linguistic Society, 292-305.

Lebsanft, Franz (2005): Kommunikationsprinzipien, Texttraditionen, Geschichte. - In: Angela Schrott, Harald Völker (Hg.): Historische Pragmatik und historische Varietätenlinguistik in den romanischen Sprachen, 25-43. Göttingen: Universitätsverlag Göttingen.

Lebsanft, Franz (2006): Sprecher zwischen Tradition und Innovation: Zum Problem von „Diskurstraditionen' und ,Diskursgemeinschaften' am Beispiel der Sprache der Politik. In: Zeitschrift für romanische Philologie 122, 531-548.

Lebsanft, Franz (2015): Aktualität, Individualität und Geschichtlichkeit. Zur Diskussion um den theoretischen Status von Diskurstraditionen und Diskursgemeinschaften. - In: Franz Lebsanft, Angela Schrott (Hg.): Diskurse, Texte, Traditionen. Modelle und Fachkulturen in der Diskussion. Göttingen: Vandenhoeck \& Ruprecht/Bonn University Press [in diesem Band].

Leech, Geoffrey (1983): Principles of Pragmatics. - New York/London: Longman.

López Serena, Araceli (2011): La doble determinación del nivel histórico en el saber histórico. Hacia una nueva delimitación del concepto de tradición discursiva. - In: Romanistisches Jahrbuch 62, 59-97.

Loureda Lamas, Óscar (2007): Zur Frage der Historizität von Texten. - In: Romanistisches Jahrbuch 58, 29-50.

Oesterreicher, Wulf (1997): Zur Fundierung von Diskurstraditionen. - In: Barbara Frank, Thomas Haye, Doris Tophinke (Hg.): Gattungen mittelalterlicher Schriftlichkeit, 19-41. Tübingen: Narr.

Oesterreicher, Wulf (2009): Aliquid stat pro aliquo. Diskurstraditionen und soziale Semiotik. - In: Ursula Peters, Rainer Warning (Hg.): Fiktion und Fiktionalität in den Literaturen des Mittelalters. Jan-Dirk Müller zum 65. Geburtstag, 57-81. München: Fink.

Pollak, Wolfgang (21988): Studien zum Verbalaspekt. Mit besonderer Berücksichtigung des Französischen. - Bern u. a.: Peter Lang.

Raible, Wolfgang (1980): Was sind Gattungen? Eine Antwort aus semiotischer und textlinguistischer Sicht. - In: Poetica 12, 320-349.

Raible, Wolfgang (1996): Wie soll man Texte typisieren? - In: Susanne Michaelis, Doris Tophinke (Hg.): Texte - Konstitution, Verarbeitung, Typik, 59-72. München: Lincom. 
Schlieben-Lange, Brigitte (1983): Traditionen des Sprechens. Elemente einer pragmatischen Sprachgeschichtsschreibung. - Stuttgart: Kohlhammer.

Schrott, Angela (2006): Fragen und Antworten in historischen Kontexten. Ein Beitrag zur historischen Dialoganalyse und zur historischen Pragmatik am Beispiel altspanischer literarischer Texte. - Habilitationsschrift, Ruhr-Universität Bochum.

Schrott, Angela (2011a): Von der Lebendigkeit der Heiligenleben. Traditionen der Dialoggestaltung bei Gonzalo de Berceo. - In: Nine Miedema, Monika Unzeitig, Franz Hundsnurscher (Hg.): Redeszenen in der mittelalterlichen Großepik: Komparatistische Perspektiven, 193-212. Berlin: Akademie Verlag.

Schrott, Angela (2011b): Die Zeiten ändern sich. Zur Verwendung des imparfait in narrativen Kontexten. - In: Romanistisches Jahrbuch 62, 137-164.

Schrott, Angela (2012): Heiligenrede in altspanischen Texten. - In: Nine Miedema, Angela Schrott, Monika Unzeitig (Hg.): Sprechen mit Gott. Redeszenen in mittelalterlicher Bibeldichtung und Legende, 107-126. Berlin: Akademie Verlag.

Schrott, Angela (2014): Sprachwissenschaft als Kulturwissenschaft aus romanistischer Sicht: Das Beispiel der kontrastiven Pragmatik. - In: Romanische Forschungen 126, 3-44.

Searle, John R. (1969): Speech Acts. An Essay in the Philosophy of Language. - Cambridge: Cambridge University Press.

Searle, John R. (1979): Indirect Speech Acts. - In: ders.: Expression and Meaning. Studies in the Theory of Speech Acts, 39-57. Cambridge: Cambridge University Press.

Spitzmüller, Jürgen/Warnke, Ingo (2011): Diskurslinguistik. Eine Einführung in Theorien und Methoden der transtextuellen Sprachanalyse. - Berlin: De Gruyter.

Stempel, Wolf Dieter (1972): Gibt es Textsorten? - In: Elisabeth Gülich, Wolfgang Raible (Hg.): Textsorten. Differenzierungskriterien aus linguistischer Sicht, 175-179. Frankfurt a. M.: Athenäum.

Togeby, Knud (1982): Grammaire française, vol. II: Les formes personnelles du verbe, publié par Magnus Berg, Ghani Merad, Ebbe Spang-Hanssen. - Kopenhagen: Akademisk Forlag.

Warnke, Ingo (Hg.) (2007): Diskurslinguistik nach Foucault. Theorie und Gegenstände. - Berlin/New York: De Gruyter.

Wierzbicka, Anna (1985): Different cultures, different languages, different speech acts. - In: Journal of Pragmatics 9, 145-178.

Wierzbicka, Anna (2010): Cultural scripts and international communication. - In: Anna Trosborg (Hg.): Pragmatics across Languages and Cultures, 43-78. New York: De Gruyter.

Wilhelm, Raymund (2001): Diskurstraditionen. - In: Martin Haspelmath u. a. (Hg.): Sprachtypologie und sprachliche Universalien. Ein internationales Handbuch. Berlin/New York: De Gruyter, Bd. I, 467-477.

Wilhelm, Raymund (2011): Die Scientific Community - Sprachgemeinschaft oder Diskursgemeinschaft? Zur Konzeption der Wissenschaftssprache bei Brunetto Latini und Jean d'Antioche. - In: Wolfgang Dahmen u. a. (Hg.): Die romanischen Sprachen als Wissenschaftssprachen. Romanistisches Kolloquium XXIV, 121-153. Tübingen: Narr.

Wrana, Daniel u. a. (Hg.) (2014): DiskursNetz. Wörterbuch der interdisziplinären Diskursforschung. - Berlin: Suhrkamp. 